\title{
Aprendizajes sobre estudios de género y desarrollo (EGED): De la sistematización a las propuestas para la mejora de las acciones en España
}

\section{RESUMEN}

Maria Estefanía Molina Bayón estefania.molina@uam.es Universidad Autónoma de Madrid (UAM), Madrid, España.

\begin{abstract}
La formación y la investigación especializada son elementos clave para la conformación de los Estudios de género y desarrollo (EGED). Los aprendizajes sistematizados servirán como herramienta clave para la promoción de la igualdad en los programas y acciones de la cooperación para el desarrollo, resultado del I y II Congreso de Estudios de género y desarrollo (ALyC-España). El análisis fortalecerá los vínculos en el ámbito de "Género, Gestión del Conocimiento y Eficacia y Calidad del Desarrollo", facilitando el diálogo político entre los distintos actores para una mayor armonización, aplicación efectiva y real de la igualdad de género y de los derechos humanos, además del empoderamiento de las mujeres en la lucha contra la pobreza hacia un desarrollo humano sostenible e inclusivo.
\end{abstract}

PALABRAS CLAVE: Estudios de género en desarrollo (EGED). Gestión del conocimiento. Aprendizajes. Armonización entre actores y eficacia y calidad del desarrollo. 


\section{INTRODUCCIÓN}

El artículo abordará los aspectos centrales relativos a algunos de los aprendizajes extraídos de una sistematización, en torno a dos congresos sobre Estudios de Género y Desarrollo (EGED)en España y América Latina y Caribe (ALyC). Es de señalar que las experiencias sistematizadas se enmarcan en el ámbito de la gestión del conocimiento bajo una perspectiva de género para la mejora de la eficacia y calidad del desarrollo. Igualmente, los congresos así como las jornadas o foros de debate son instrumentos que dentro de la Cooperación Universitaria al Desarrollo (CUD) con perspectiva de género, se adscriben al bloque temático de Estudios y Sensibilización (la tipología en torno a los diferentes bloques temáticos en Cooperación Universitaria al Desarrollo (CUD) con perspectiva de género, aparece reflejado en "Consideraciones en torno a la Cooperación Universitaria al Desarrollo con perspectiva de género. En Molina E, San Miguel N (2009). Nuevas Líneas de Investigación en Género y Desarrollo. Cuadernos Solidarios, Ediciones UAM, Madrid). A partir de lo mencionado, lo expuesto tendrá como finalidad contribuir a través de unas propuestas, en primer lugar al reconocimiento y consolidación de los EGED y seguidamente al fortalecimiento institucional de la CUD con perspectiva de género.

\section{LA SISTEMATIZACIÓN COMO INSTRUMENTO DE GESTIÓN DEL CONOCIMIENTO AL SERVICIO DEL DESARROLLO}

Según señala Oscar Jara (1998), el concepto de sistematización de experiencias ha sido creado históricamente en América Latina como producto del esfuerzo por construir marcos propios de interpretación teórica, desde las condiciones particulares de la realidad (JARA, 1998). El autor enuncia que esta herramienta puede ser considerada como una interpretación crítica, atravesada por un proceso de reflexión en torno a una o varias experiencias, las cuales posibilitarán, por medio del ordenamiento, clasificación de información y reconstrucción, la constitución de líneas de debate o investigación. Dicho de otra manera, sistematizar bien podría ser entonces reconstruir experiencias, analizar e interpretar críticamente lo ocurrido para llegar a profundizar y comprender lo analizado. Por tanto, la sistematización, alude a la extracción de aprendizajes basados en una interpretación crítica de la lógica integral de experiencias, reconstruyendo sus procesos y contenidos (VAN DE VELDE; JARA, 2002). A partir de estas afirmaciones, la estructura de nuestra sistematización seguirá un camino trazado y dará contenido a unas respuestas, en relación a unos principios básicos diseñados.

Así, desde un punto de vista teórico, ésta abordará las siguientes preguntas:¿Para qué sistematizar?; ¿Qué experiencias y en qué lugar y periodo de tiempo sistematizar?¿Qué aspectos centrales de la sistematización se adecuan más al contexto al cual se adscriben? y por último, se confluirá en el horizonte o muestra de los aprendizajes que permitan experimentar la mejora de las acciones a futuro. De este modo, se muestra aquí, una visualización de los puntos departida, parada y final de la sistematización, con el propósito de brindar el hilo conductor de la misma, a partir de la cual se han obtenido los aprendizajes a presentar. El punto de partida es la descripción narrativa de todas y cada una de las intervenciones en las mesas redondas, paneles y conferencias que han 
conformado los dos congresos de EGED, las cuales por cuestión de espacio nopueden ser desarrolladas en este artículo. Posteriormente, el punto de paradacorrespondiente a la extracción de aprendizajes dará como resultante, la apertura de líneas de investigación a debate para finalmente llegar a una serie de propuestas a modo de recomendaciones.

Se ha de añadir que esta sistematización ha sido trabajada desde la forma de relato narrativo tanto descriptiva como interpretativa, combinando ambos enfoques. A saber: el enfoque de casos, bajo el cual se capturó y registró diversas experiencias consideradas exitosas e inclusivas, con el objetivo de que puedan ser un referente y así facilitar su replicación; y el enfoque de temas, cuya motivación fue originada a partir de la búsqueda de la facilitación del diálogo político entre los distintos actores del desarrollo -especialmente con las instituciones de educación superior- para una mayor armonización de la igualdad de género en los procesos de desarrollo. Además, también para el fortalecimiento de los vínculos entre "Género, Gestión del Conocimiento y Eficacia y Calidad del Desarrollo", lo cual contribuirá al empoderamiento y la aplicación efectiva y real de los derechos de las mujeres en la lucha contra la pobreza hacia un desarrollo humano sostenible e inclusivo. Y finalmente el punto de llegada, que consistirá en recoger los frutos de la cosecha, que se traducirá en una serie de lecciones o recomendaciones derivadas de los aprendizajes.

\section{CONTEXTO: SISTEMATIZACIÓN DEL I Y II CONGRESOS DE ESTUDIOS DE GÉNERO Y DESARROLLO (EGED) EN AMÉRICA LATINA Y CARIBE (ALYC) Y ESPAÑA.}

El primero de los congresos sobre EGED, fue celebrado en España (Madrid) 2009 en la sede de la Universidad Autónoma de Madrid (UAM) coordinado por la Red GEDEA (Género en Desarrollo y Eficacia de la Ayuda). Y el segundo, en el 2012 en República Dominicana (Santo Domingo)bajo la co-coordinación del Centro de Formación y Capacitación de ONU Mujeres y la Red GEDEA. De ambas experiencias, se reflexiona en torno a la gestión del conocimiento y la CUD con perspectiva de género, extrayendo aprendizajes generados desde la sistematización de los mismos, hacia la puesta en práctica de estudios de género para las políticas de desarrollo e igualdad en el estado español y en los países de la región latinoamericana.

La necesaria y buscada sistematización responde en buena medida, a una demanda por parte de la institución responsable de la planificación y evaluación de políticas de desarrollo, ex DGPOLDE. En este sentido, nos hemos de trasladar al 2010, fecha en la que se inició un proceso de diálogos políticos entre actores y fortalecimiento de investigación-acción y de capacidades financiado y apoyado por la Cooperación Española (CE).

Por su parte, ONU Mujeres como Organismo Internacional con mandato de género, tambiéntrabajó para la igualdad de género y el desarrollo de la mano de la Eficacia de la Ayuda (EA), a través del proyecto "Apoyo al monitoreo de la Eficacia de la Ayuda desde una perspectiva de género" adscrito al ex INSTRAW. En este sentido, el Centro de Formación y Capacitación en género situado en República Dominicana, comenzó la búsqueda de alianzas para la EA, con los diferentes actores y entre ellos, concretamente con los especializados en formacióncomo lo eran y aún lo siguen siendo las universidades, centros de 
estudios, Redes de investigación y capacitación de países de la región latinoamericana y del estado español.

\section{MARCO TEÓRICO Y JUSTIFICACIÓN}

El recorrido histórico de los processos formativos académicosen ámbito de Género y de Desarrollo reflejauna enorme complejidad que mayoritariamente ha ido acompañados de múltiples dificultades en elcontextointernacional. En lo que respecta a la enseñanzaacadémica, se puede afirmar quedesde la última década de finales de los años50, se pon em las bases para la articulación futura de los EGED. Durante este periodo y principalmente des de las universidades norte americanas, se difunden tanto los primeiros cursos de estúdios de Género (los primeros cursos de estudios de género, fueron inicialmente definidos como Estudios sobre la Mujer.

A partir de mediados de los 70 ,los estúdios feministas introducen el concepto de género, de la mano de investigadoras anglosajonas como Gayle Rubiny Joan Scott) como los de desarrollo. Estos últimos, destacan en el campo de las relaciones internacionales, mientras que losrelativos a lasmujeres lo hacen por el creciente processo de protagonismo e incidência del movimento feminista em la sociedade civily también em las universidades.

Los EGED nacidosdes de estos dos caminhos separados, em cuentran su intersección a partir de la visibilización de la mujer em los processos de desarrollo a media dos delos años 70, sobre la base del enfoque MED (Mujeres em Desarrollo). Estos se irán introduciendo em las principales Agencias Internacionales de Desarrollo, sobre todoporlasprofesionalesfeministas que trabajan en estas instituciones. $Y$ se presentanen un principio como processos formativos no oficiales, orientados a la promoción de competências prácticas em elámbito del Desarrollo, vinculados principalmente a actores sociales em el marco dela Cooperación internacional, como los movimentos feministas, organizaciones no gubernamentales y Agencias de Desarrollo.

Otro de los factores que ha de ser mencionado en relación a la consolidación de los EGED, es la apertura del debate mundial sobre la condición de la mujer y su lugar privilegiado em la agenda internacional, favorecido por um conjunto de eventos que se em marcan em la denominada Primera Década de las Naciones Unidas para la Mujer (1975-1985). Em 1975 se celebra la I Conferencia Mundial sobre la Mujeren México y posteriormente, em 1979, la Convención sobre la Eliminación de todas las Formas de Discriminación contra la Mujer(CEDAW), como intento concreto de reconocer la discriminación hacia las mujeres como uma violación de los Derechos Humanos. A partir de ella, se adoptan medidas obligatorias para la erradicación de dicha discriminación en todas las esferas de la vida, entre otras, en la de la educación.

Concretamente en 1985 se celebra la Tercera Conferenciaen Nairobi, donde aparece por primera vez el concepto de Género, aun que desvinculado de aplicación práctica de las políticas de desarrollo. Y es a raíz de esta conferencia, cuando em pieza na evidenciarse las primeras iniciativas académicas a islãda sem el ámbito de los EGED. En el estado español por ejemplo, destaca el Curso de Especialización en Mujery Desarrollo promocionado por el Instituto de la Mujer, cuy a primera edición se lleva a cabo em 1990. Pero no es hasta1995 com la 
Cuarta Conferencia Mundial sobre la Mujer, em Beijing cuando el "género" pasa a ser prioritário em el ámbito de la Cooperación al desarrollo desde un punto de vista teórico, a través del reconocimiento del enfoque GED (Géneroen Desarrollo).

A nível práctico y de estrategia de acción, se planifica la aplicación de la Plataforma para la Acción de Beijing, estructurada em 12 áreas de actuación prioritarias con objetivos comunes, entre los que se encuentra la educación. Este processos upuso la concreción de dos estrategias: la transversalización del enfoque de Género em las dimensiones tanto teóricas como prácticas del Desarrollo y elempoderamiento de las mujeres para incrementar SUS capacidades y fortalezas. Em lo que conciernea los EGED, la Plataforma evidencia explícitamente medidas para su promoción em la educación superior, obteniendo como resultados la multiplicación de acciones formativas acadêmicas de posgrado em los diferentes Estados signatarios.

Así, em el área dedicada a los derechos de mujeres y niñas em la enseñanza, el documento de la Plataforma propone (LEY RAB; DE LA FUENTEP; ORTEGAR (2009): "Apoyary realizar estúdios e investigaciones sobre el género em todos los níveles de la enseñanza, especialmente em el nível depostgrado em las instituciones académicas, y aplicarlos a la elaboración de programas, incluídos losde estúdios universitarios..." y "Elaborar programas de educación em matéria de derechos humanos que incorporen la dimensión de género em todos los niveles de la enseñanza, em particular fomentando la inclusión em los planes de estúdio de las instituciones de enseñanza superior, especialmente em los planesde estúdios de postgrado em los campos jurídico, social y de ciências políticas, del estúdio de los derechos humanos de la mujer tal como figuran em las convenciones de las Naciones Unidas".

Posteriormente, com la revisión de los objetivos de la Conferencia de Beijing em 2000 y 2005 se refuerza la necesidad de las estrategias planteadas de transversalidad del enfoque de género y empoderamiento de las mujeres, posicionando a los processos formativos em GéneroyDesarrollo (GED)como grandes contribuyentes a las mismas.

Así, por medio de la transversalización se prioriza y fortalece la integración de la perspectiva de género en la educación tanto em las experiências formativas como em los processos investigadores en GED. Por tanto, se puede afirmar que durante las últimas décadas, más en concreto desde que se celebra la IV Conferencia de la ONU sobre las Mujeres en Beijing, se produce un impulso fundamental para favorecer políticas de igualdad que promueven avances en la mejora de la situación de las mujeres, como actoras de derechos y constructoras de democracia, desarrollo y paz. En este proceso de institucionalización de políticas públicas contra la discriminación hacia las mujeres, surge la necesidad de profundizar en la investigación, el conocimiento y consiguientemente en los EGED.

Al mismo tiempo comienzan a aparecer iniciativas formativas y de estudios de género que facilitan a las mujeres sensibilizadas o activistas desde el feminismo, la construcción de teorías y prácticas para integrar el enfoque de género de manera sistemática y concienciada en los diferentes ámbitos desde los que participan, como en la política, la sociedad o la economía etc. En concreto, en España como se ha mencionado, se refuerzan y visibilizan iniciativas 
formativas específicas de estudios de las mujeres que ya existían y aparecen los primeros master y posgrados de políticas de igualdad o de relaciones de género y desarrollo durante toda la década de los 90 . Por otro lado, en ALyC se produce también un impulso importante de estas temáticas en algunas universidades o instituciones vinculadas a la educación, a la vez que desde las organizaciones de mujeres y feministas se multiplican iniciativas formativas no regladas, así como de investigación sobre género y posgrados que vienen en muchos casos financiadas por las cooperaciones internacionales presentes en la región.

Una vez enunciadas algunas pinceladas en torno a los orígenes y basamento de los EGED, se ha de subrayar su desarrollo a partir del año 2005vinculadosa la Agenda de la Eficacia de la Ayuda (EA). En esta fecha se concreta esta misma, produciéndose un punto de inflexión, que se traduce en una transformación sin precedentes en las políticas para el desarrollo mundiales, con un impacto de gran magnitud en el contexto político, social, económico, cultural y por ende en el educativo.

Este último cobra un interés especial por la temática a tratar, máxime bajo la influencia de un mundo en crisis, derivándose una necesidad imperante de nuevos paradigmas en la propia concepción de las políticas de cooperación internacional en su acción multilateral y bilateral, además de en la concentración de la Ayuda y la consiguiente priorización geográfica. Por todo ello, el papel de la Academia y con ella, los aprendizajes a extraer de los EGED en cuanto a democratización del conocimiento y fortalecimiento de la CUD con perspectiva de género, adscritos a la mejora de la gestión del conocimiento, resultan fundamentales a la hora de tomar decisiones estratégicas de actuación para la igualdad de género hacia 2015 y 2020.

Así, el intento de ser más eficaces, habrá de pasar partiendo de los aprendizajes ya comprobados como exitosos y hacia la preservación y defensa de los derechos humanos para la igualdad en las Políticas de Desarrollo. Por otro lado y en relación a la eficacia deseada, también es significativo señalar que después de años de trabajo y cabildeo del movimiento de mujeres en todo el mundo para que la ayuda supusiera un cambio real en la vida de las mujeres, no es hasta 2008 con la Agenda de Acción de Accra, cuando se logra hacer explícita la necesidad de construir el desarrollo sobre el paradigma de derechos humanos y el enfoque de género.

Aunque esto, no supuso la eliminación de las dificultades para obtener la plena participación de las organizaciones y movimientos de mujeres, ni se palió la falta de mecanismos de participación e indicadores para la evaluación de impacto de la Ayuda para el Desarrollo en las mujeres. Y por lo que concierne al marco universitario en el estado español, aun que existen documentos de referencia normativos, que intentan consolidan el compromisso de las instituciones de educación superior em el ámbito de la CUD con perspectiva de género, el processo de integración y sinergias entre las políticas de igualdad y las de desarrollo em la política educativa universitária no está exento de desacuerdos, evolucionando a ritmos distintos y muy lentamente.

Existen muchas debilidades en el ámbito de GED sobre todo institucionales, por lo que los EGED suponen un apoyo y fundamento para la investigaciónacción, el establecimiento de diálogos políticos, fortalecimiento de capacidades etc. y en definitiva para la consolidación de la CUD con perspectiva de género. $Y$ 
es que, la experiencia de múltiples iniciativas durante los últimos 20 años, han demostrado que la formación y los estudios de género son una de las herramientas más efectivas para avanzar hacia la igualdad en contextos de transformación democrática. La formación, los estudios y la investigación especializada en GED son por tanto, elementos indispensables y concretos para el fortalecimiento y empoderamiento de las mujeres en la lucha contra la pobreza.

El momento actual, a través de los análisis, informes y estadísticas, revelan que el logro de la transversalidade del género y el empoderamiento de las mujeres está cada vez más alejado, máxime en contexto de crisis económica, junto a las recientes muestras de retroceso en los derechos de las mujeres. En consecuencia, la igualdad de género em el ámbito del Desarrollo dista mucho de ser uma prioridade objetiva para las universidades o instituciones de educación superior, por lo que, decara tanto a los compromissos internacionales como a la agenda política nacional del estado español y de los países de ALyC para la consecución de uma igualdad de género, se hace patente la necesidad del fortalecimento y consolidación institucional para uma real integración del género em los processos de CUD y democratización del conocimiento.

\section{METODOLOGÍA Y ESTRUCTURA}

Los dos congresos se estructuraron alrededor de ponencias organizadas por mesas temáticas, conformadas por profesionales y expertas del mundo académico, de la sociedad civil, así como de instituciones gubernamentales nacionales e internacionales. Cada mesa temática fue orientada por una persona que moderó, introdujo a cada conferencista o participante y gestionó el turno de preguntas del público a modo de cierre y conclusiones. Sintetizando, se puede decir que la dinámica de ambos congresos se basó en paneles y mesas redondas, para seguidamente abrir la discusión a las participantes. Se ha de señalar, que los turnos de preguntas y aportaciones permitieron compartir experiencias y discutir ampliamente los temas expuestos por las panelistas, así como identificar y desarrollar nuevas estrategias y metodologías para la incidencia política y la democratización de conocimiento y los saberes.

Ambos congresos fueron co-coordinados por la Red GEDEA y tanto las personas participantes como los temas a tratar fueron guiados por unos objetivos vinculados a la gestión del conocimiento sobre los EGED como factor de transformación y de desarrollo. La obtención de los resultados, se basó en la compilación de las reflexiones y las discusiones llevadas a cabo durante los dos congresos, así como en las propuestas de expertas dirigidas hacia la planificación, ejecución y evaluación de acciones planteadas. El enfoque adoptado fue fruto de la combinación del de casos y temas, por lo que fue muy importante capturar y registrar experiencias diversas, no sólo procedentes de las universidades o centros institucionalizados, sino también de la sociedad civil y en concreto de las mujeres en exclusión social, mayoritariamente discriminadas y no consideradas sujetos de conocimiento, como mujeres indígenas, afrodescendientes, campesinas etc.

Se seleccionaron experiencias exitosas e inclusivas en el marco de los EGED, 
nivel regional. $Y$ en cuanto a los temas, se procuró focalizar en aquellos que respondían a la motivación originada a partir de un tema adscrito a la gestión del conocimiento y la EA, como fuela gestión y democratización del conocimiento desde una perspectiva de género para la Eficacia y calidad del Desarrollo.

\section{OBJETIVOS}

Previo a la celebración de los dos congresos de EGED, se produjo una identificación de algunos aspectos claves para los EGED, susceptibles de ser objeto de análisis por sus efectos en el desarrollo y en el empoderamiento y aplicación real y efectiva de los derechos de las mujeres. Entre estos se encuentra, el impacto del conocimiento y los saberes de las mujeres, en la lucha contra la pobreza. De igual modo, también resultaría muy dilucidador visibilizar el papel de los EGED en la formación de recursos humanos especializados en las políticas GED, junto al énfasis en la integración del enfoque de género en la formación y en el sistema educativo en su conjunto, en concreto en la educación superior y especializada.

Pero también se habría de tomar en cuenta, los conocimientos, experiencias y estudios de género desde sistemas informales, de la mano de organizaciones de mujeres y movimientos feministas, como creadores de teorías y prácticas feministas para la defensa de los humanos de las mujeres. El análisis debía incluir no sólo a la Academia, sino también a todo el movimiento feminista en sus múltiples manifestaciones, como ONGD, asociaciones, plataformas etc. Por tanto, otro de los planos a focalizar habría de ser el fortalecimiento de sinergias y pactos para la igualdad de género y búsqueda de alianzas con diferentes actores especializados en la formación como universidades, centros de capacitación, investigación de OOII, ONGD etc.

A continuación se muestran de manera resumida algunos de los objetivos comunes a los dos congresos:

-Contribuir a posicionar el tema de la gestión del conocimiento y la CUD con perspectiva de género en la Agenda de la Eficacia y Calidad del Desarrollo y difundir sus avances.

-Visibilizar el impacto de los EGED y su función en los procesos y políticas de desarrollo y en el cumplimiento de los derechos humanos de las mujeres. Así como difundir experiencias exitosas en el ámbito de la formación, al igual que el papel de las Redes de conocimiento, investigación o incidencia en GED.

-Dar cuenta de la relevancia en torno a la retroalimentación y relación entre el mundo académico y el activismo del movimiento feminista en sus diversas expresiones entre estado español y ALyC.

- Fomentar el desarrollo de procesos de democratización del conocimiento y diálogo de saberes entre los distintos actores del Desarrollo, especialmente entre instituciones de educación superior como universidades con centros de estudios o investigación de ONGD, OOIl etc.

-Contribuir a la búsqueda de información y financiación para la actualización y sostenibilidad de los EGED en relación a la Agenda de Desarrollo, las nuevas modalidades de la Ayuda, en un contexto de crisis económica y el retroceso de las políticas de igualdad y desarrollo. 


\section{APRENDIZAJES EN EGED: LÍNEAS DE INVESTIGACIÓN A DEBATE Y RECOMENDACIONES PARA LA MEJORA DE LAS ACCIONES}

Como ya se ha mencionado de manera introductoria, ambos congresos estuvieron nutridos por una participación amplia y diversa de universidades, centros de estudios, departamentos de investigación y capacitación de ONGD, sociedad civil y expertas en GED de Organismos nacionales e internacionales. La sistematización facilitó que todos estos actores, se involucraran en los procesos de aprendizaje para la generación de nuevos conocimientos e iniciativas a modo de líneas de debate y posterior recomendaciones.

Estos aprendizajes están destinados a que todos los actores de Desarrollo, puedan apoyarse para planificar en la confluencia del marco de Igualdad de género y democratización del conocimiento. $Y$ también para que posibilite la ejecución en base a toma de decisiones derivadas de unas propuestas de acción en EGED extensibles a la CUD con perspectiva de género.Es decir, el siguiente apartado básicamente apuntará, a partir de las experiencias descritas y sintetizadas, como aprendizajes articulados en bloques temáticos que a modo de líneas de investigación a debatirdarán lugar a propuestas de accióno recomendaciones, para la mejora de la gestión del conocimiento bajo una perspectiva de género.

\section{LÍNEAS DE INVESTIGACIÓN A DEBATE PARA LA DEMOCRATIZACIÓN Y EFICACIA DEL CONOCIMIENTO EN LOS PROCESOS DE DESARROLLO.}

A continuación se expondrán de manera sintética una serie de líneas de debate, derivadas de los aprendizajes junto a la identificación de los retos más significativos, extraídos del I y II Congreso sobre Estudios de Género y Desarrollo (EGED):

\section{LOS EGED: ENTRE EL PENSAMIENTO FEMINISTA Y LA TECNOCRACIA DE GÉNERO}

Es necesario, señalar de los EGED la vinculación entre el pensamiento feminista académico y el de los movimientos sociales de mujeres. La influencia de estos últimos, há sido sin duda, uma cicate para su evolución, pues los inicios de la disciplina estu vieron vinculados a la reivindicación feminista, encontra del sés goandro céntrico de las ciências sociales. Esta demanda junto a las aportaciones de las mujeres em la producción científica fue contemplada em la agenda internacionalde mujeres em el desarrollo.

Este objetivo suponía la de construcción del conocimiento dominante, para luego proceder a la fase de recuperación, reconstrucción y reflexión. Así, los EGED fueron construyendo su propia metodología, teoria y categorías, respondendo a la necesidad de la disciplina y al intento de recuperar datos cuantitativos y cualitativos desde una perspectiva degénero em los contextos del desarrollo.

Em los últimos años, y sobre todo a partir de los años 90, los EGED há nesta dos ometidos a um processo de reflexión, caracterizado por la auto-conciencia y auto-critica. Las nuevas teorías y el cambio del paradigma de enfoque MED al GED em las políticas y las prácticas del desarrollo, hangenerado nuevas 
expectativas, pero también retos associados com la evolución del conocimiento en GED, junto a su aplicación práctica. El proyecto de la transformación social de gênero requería uma sintonia em trelos avances teóricos y elactivismo, además de uma fuerza política para exigir el cumplimiento delos compromissos internacionales y estatales com los derechos humanos de las mujeres.

No obstante, los esfuerzos no siempre hantraído los resultados esperados, pues los procedimientos de gênero y desarrollo utilizados para acelerar los cambios, en ocasiones, pasaron a ser uma fuente de decepciones. Em este contexto, el enfoque GED há sido criticado por conformarse como uma categoria tecnocrática, por su funcionalismo en relación al poder existente, así como por mostrar poco interesse nuna modificación real de las desigualdades.

Su tecnificación e instrumentalización, há supuesto asimismo el reducionismo y la simplificación de las categorías, convirtiéndo las em unos conceptosa-históricos, apolíticos y descontextualizados, dejando intactas las relaciones de poder predominantes y desiguales. En este sentido, la despolitización dela categoria de género há aberto um debate sobre la diferencia entre "hacergénero" y "hacerfeminismo" eneldesarrollo, aunque este en realidad, se inscribe em una reflexión más amplia sobre la despolitización y la repolitización de los objetivos feministas em el desarrollo.

Sin embargo y aunque la tecnificación del enfoque mencionado, se presenta como un instrumento útil, menos reflexivo y autocrítico, la experiência muestra, que lo que en parte, marca ladiferencia en los procesos o acciones, son las convicciones feministas de quienes las ejercen, las cuale simplican um compromisso personal que finalmente promueve el cambio.

Por otro lado y desde esta perspectiva, los EGED pueden interpretarse como um brazo acadêmico del feminismo y de los movimentos demujeres, en la contribución de la concienciación y transformación social a favor de los derechos de las mismas. De ahí, que su incidência no sólo sea notoria em la producción y em la difusión del conocimiento, sino también em la formación de lo sagentes del cambio. Por ello, se reivindica uma academia militante, que conecte com los problemas sociales y se implique em los processos de transformación a favor de la igualdad de género.

La contribución de las universidades y otros centros de EGED no sólo consiste em la investigación aplicada, la formación y la difusión del conocimiento, sino también en acciones de sensibilización que fomenten debates y nuevas líneas y espacios de reflexión desde um pensamento crítico, permitiendo de esta manera incentivar la implicación acadêmica y el compromisso social com los valores de la igualdad de gênero y la justicia social.

\section{DESCOLONIZACIÓN DEL CONOCIMIENTOY DESARROLLO: DIVERSIDAD, DIÁLOGO FEMINISTA Y DE SABERES COMO ELEMENTOS CONSTITUTIVOS DE LOS EGED}

Los EGED dentro y fuera de la academia muestran múltiples conexiones com el poder. Por ello, es necesario abordar la conexión inevitable de estos con la cultura, debate desde el cual se han generado numerosas críticas relacionadas com el desarrollo y la práctica de la cooperación internacional. Escobar (1984) hande mostra do que los interesses políticos que están de trás de la ayuda al desarrollo tienen un impacto em los proyectos, reproduciendo las desigualdades 
de poder, no solo por la ideologia que atraviesa la acción del desarrollo, sino también por la misma manera dellevar la acabo. Y por otro, se ha mostrado el poder del conocimiento y la reivindicación de la descolonización del pensamento y de la propia academia, com el fin de abrir nuevos espacios del saber y del ser, desde el reconocimiento de valores, saberes populares y prácticos de outros pueblos y culturas no-occidentales.

En relación a la cultura, algunas teorias del desarrollo Sem (1999) reconocen que los valores culturales juegan um papel significativo em la formación de las capacidades delas personas para cambiar sus vidas y las sociedades em las que viven. No es menester olvidar, que la cultura em simisma está atravessada por las relaciones de poder y los câmbios cultural estienen quever com las negociaciones y re-negociaciones del poder.

Como ya se ha anunciado, estas juegan um papel importante em la formación de las capacidades de la comunidade y em los processos associados a la transformación social. Los sistemas cultural ES pueden conferir también el poder y los privilégios a um grupo social determinado y negarlos derechos y el acceso a los recursos aotros, lo que se explica em la teoria de gênero respecto a las relaciones de desigualdade entre hombres y mujeres. Además, se reconoce que cada intervención al desarrollo com lleva câmbios culturales y éstas tienen sus implicaciones em las negociaciones y renegociaciones de las identidades de género, afectando a la posición que ocupan las mujeres em la sociedad.

El pensamento y la práctica del desarrollo no son neutros, si no que están cargados de valores y suposiciones culturales y las personas que trabajan em el desarrollo actúan según sus propias percepciones, construidas muchas veces em base a los prejuicios y estereotipos. Estas percepciones pueden influir em las decisiones y las acciones del desarrollo y están influenciados por las dinâmicas de poder que se esconden de trás dela categorización del mundo. Dado que cada intervención para el desarrollo implica cambios, ES especialmente importante tener em cuenta y desafiar constantemente dichas suposiciones culturales, junto a sus dinámicas, para que el impacto del desarrollo sea más sensible y consciente, además de consenso adoy protagonizado por la población local.

La cooperación para el desarrollo es um processo em dos direcciones, que debe implicar el aprendizaje y el conocimiento mutuo. Um cuidado so análisis de los contextos del desarrollo, y especialmente de las relaciones de poder inherentes a la cooperación y a los sistemas sociales, tiene um valor enorme para la cooperación al desarrollo y em particular para el trabajo en el ámbito GED.

De igual modo, los EGED juega nun papel essencial en este proceso y proporcionan conocimiento, al mismo tiempo que son fuente de su renovación, através de descolonización del saber y serenla teoria y práctica. Por ello, es vital aprovechar el potencial de la investigación aplicada, permitiendo enfrentar los desafios culturales y diluir las fronteras artificiales entre la práctica y la teoria del desarrollo, para avanzar em proyectos conjuntos, com el fin de poner em práctica la ética de los derechos humanos y la igualdad de género.

Por tanto, las reflexiones sobre la descolonización de la academia y del conocimiento, reflejadas en los EGED, quedan recogidas como reto em el mundo diverso de hoy, donde la interacción com otros pueblos, culturas y modos de 
vida, hace necesario repensar la historia y el pensamento dominante. Especialmente desde ALyC y en concreto desde las voces de representantes indígenas, la diversidade empezó a hacerse patente, sobre todo por su conexión com las cuestiones étnicas y culturales, pero sin limitarse a ellas. Se su brayó la necesidad de abordar el debate cultural dentro del feminismo desde uma posición de respeto y escucha hacia otras mujeres, lo que brindó reconocimiento a los feminismos culturales y post-coloniales.

La implicación más participativa de las mujeres de los grupos marginados em la producción científica, empezó a entenderse no solo como conocimiento e investigación académica, sino también como saberes basados em las abiduría desde lo vivencial y lo cotidiano. Por ello, se considero especialmente importante crear oportunidades para las mujeres indígenas en lo que respecta al acceso a las universidades y poder prosseguir uma carrera acadêmica e investigadora.

Hasta el momento, la producción científica generada por los pueblos indígenas es escassa y mucho más por las mujeres indígenas, pues lo habitual es que se na otras mujeres las que hablan por ellas y su historia. E neste contexto las mujeres indígenas reivindican, no ser tratadas como meros objeto de estudio, receptoras de fondos en proyectos o políticas, sino autoras de los mismos y hablar con autoridad de su propia experiencia y saberes desde múltiples espacios, entre los que también se encuentra la academia.

Contemplar la diversidad desde sus múltiples aristas, implica uma relación compleja com el feminismo, lo que a su vez replantea entre otras cuestiones, el objetivo de la igualdad desde la diferencia y la heterogeneidade de la identidad sexual. También es esencial, reflexionar en torno a como la diferenciación del gênero se conecta com otras divisiones e identidades como la etnicidad, la sexualidad, la edad,etc. Además de cómo se associa com las relaciones de poder em los sistemas dominantes.

A esto hay que añadir el impacto em la política y la práctica de gênero y desarrollo, de la perspectiva de las identidades múltiples y dinámicas, fluidas y em construcción constante. E neste contexto se subraya, el apoyo en la promoción de mecanismos de investigación y conocimiento para entender mejor la diversidad y aprender de esta experiencia, em diálogo com otras mujeres.

En este sentido, se hace referencia al aprendizaje y establecimiento del diálogo entre las mujeres em su diversidad y búsqueda de alianzas para el fortalecimiento del conocimiento em la práctica en GED. Por tanto, se evidencia el reconocimiento de las mujeres em su diversidade y diferencias hacia la construcción de consensos comunes, através del tejido de redes, solidaridad y cooperación mutua.

Así, los consensos se presentan como esenciales, sobre todo entre las organizaciones de mujeres, pues aunque de difícil logro, se considera imprescindible elapoyo como primer paso, para posteriormente identificar y partir de lo común hacia la construcción de nuevos saberes, líneas de investigación y prácticas en GED. 
El intercambio de experiências entre las universidades latino americanas y españolas muestra que existen retos em común, al menosen lo que se refiere al a institucionalización y evolución delos EGED. Estos se vinculan especialmente com elg rado de transversalidade degénero, em las universidades em distintos niveles. A saber: en la CUD en su conjunto y específicamente em su organización y gestión interna; em lacre ación y transmisión del conocimiento, así como em la proyección externa de las universidades.

Paradójicamente, la producción teórica de las acadêmicas feministas, no siempre há estado acompanhada por la aplicación práctica de este conocimiento em la política y cultura organizacional delas instituciones de educación superior. Ello se debe en gran parte, a las resistências y reproducción delas desigualdades de género em la propia estructura, siendo esta tendencia común em todas las partes del mundo. Hay evidencias que muestran la presencia creciente delas mujere sem las universidades, como alumnasy profesoras, mientras que se observan la ausencia de cambios sustanciales em los órganos de decisión y de poder, siendo éstos ocupados casi en su totalidad por hombres.

Hay pocas catedráticas, rectoras, decanas, jefas de departamentos o de centros de investigación, como resultado de la dificultad en la promoción delas mujeres em la academia, derivada por ejemplo, de la pervivencia deses gossexistas em la composición de tribunales de evaluación y en la concesión de proyectos de investigación. $Y$ es que, apesar de los avances em las políticas públicas a favor de la igualdad de género, todavia que da mucho por haceren la puesta em práctica de la legislación y las declaraciones institucionales en el ámbito académico.

No solo hace falta uma mayor paridade em las estructuras de poder universitario, si no también ES necessário asegurar que las mujeres que entren al poder, tengan uma preparación adecuaday sensibilidade algéneroen representación de los interesses del resto de mujeres. Por ello, se requiere formar líderes políticas y acadêmicas de recambio, con la finalidad de ocuparlasposicionesydar continuidad altrabajodesusprecursoras.

Los processos y procedimentos de trabajo que inciden em el avance de la igualdad se abren camino muy lentamente, ya que persisten las brechas entre el nivel declarativo y los câmbios reales em la cultura organizacional de las universidades. Aún, permanece uma barrera estructural e invisible en lo que se refiere a la igualdad de género real y efectiva y se mantiene uma división sexual del trabajo y de los roles estereotipados de género que influyem em la elección de carreras universitárias y em el desarrollo profesional, uma vez finalizados los estudios.

Así, suelen estar feminizadas, las ciências sociales y humanísticas, mientras que las carreras técnicas, tecnológicas y de ingeniería, masculinizadas. Los estúdios de géneros e consideran um área deconocimiento feminizado y poco atractivo para loshombres, con es caso capital simbólico, lo suficientemente prestigioso y con poder en el ámbito científico. Además, se cuestiona el valor académico o denominación de "ciencia", haciendo alusión a la falta de "objetividad" debido a una ninguneada e infravalorada metodologia de investigación feminista.

Por tanto, la categoria de género no aplica comoun elemento imprescindible y transversala tener encuenta em todas las disciplinas y âmbitos de investigación 
académica, apesar de las declaraciones y compromissos políticos que lo exigen. Em consecuencia, los estúdios de género, por lo general, adolecen de un fenómeno de "guetización", que les convierte en un área de conocimiento aislado y feminizado,con un impacto bastante limitado en las distintas disciplinas, apesar de su relevancia y del debate feminista aludido.

De igual manera es patente la inquietud que suscita la distancia y en ocasiones la oposición entre el mundo académico y el movimiento feminista. Por ello, se redunda en la idea de construir puentes entre ambos para el enriquecimiento y retroalimentación de los EGED. La superación de esta barrera a través de la creación de espacios de formación, trasciende y transforma los propios feminismos como movimientos políticos.

En este sentido, se promueve una simbiosis en la que se incorporen los conocimientos adquiridos en el "terreno" de la mano de las organizaciones de mujeres o movimientos feministas, junto al bagaje científico producido por la Academia. Otra de las claves a las que se alude es la de tejer alianzas entre los distintos actores para favorecer la negociación cultural y los diálogos de saberes, para la generación de nuevo conocimiento inclusivo en cuanto a diversidad, para así disminuir progresivamente las brechas y distancia entre Academia y sociedad.

En síntesis, se recomienda una "Academia militante" cuyo currículo de programas en torno a los EGED, se sustente en un enfoque de gestión por resultados y retroalimentación entre producción científica y activismo político. En cuanto a la ofertaformativa tanto em los estúdios degénero, como en los EGED se evidencia un gran desequilibrio y escasez, pues ambasespecialidades, son destacables a nível depostgrado, mientras que prácticamenteson inexistentes em lasbases o formación de grado a través de diplomaturasylicenciaturas.

Además, también em los planes de estúdio vigentes de las universidades españolas y latinoamericanas, se identifican dichos estudios, a través de matérias optativas, consideradas de segunda categoría. De esto se deriva, la imposibilidad de iniciar en el campo de la investigación, el ámbito de conocimiento en género y desarrollo. Raras veces se llevan a cabo investigaciones, tesis doctorales o Proyectos fin de Carrera (PFC) vinculados a esta cuestión y mucho menos publicaciones.

Se ha de señalar que em la clasificación de número estándar internacional delibro (ISBN), no queda recogida uma categoria específica que haga referencia al ámbito de conocimiento relacionada con los estudios de gênero y mucho menos con los EGED. Por consiguiente, la falta de reconocimiento y transversalidad em las instituciones de educación superior españolas y latinoamericanas, hacen que los EGED acaben ocupando un lugar voluntarioso y de interés especializado. $\mathrm{Ni}$ que decir tiene, que este es cenário dificulta el desarrollo de uma Carrera acadêmica e investigadora em el ámbito de conocimiento señalado, por lo que se plantea la necesidadde crear instrumentos ad hoc, como observatórios de gênero dentro de las universidades y específicamente de género y desarrollo.

Esto facilitaría la puesta en marcha de programas de evaluación y seguimento de las políticas universitarias, así como el monitoreo de programas y acceso a la información sobre el impacto específico de los EGED. Así, la información sobre las trayectorias profesional ed de los y la salumnas de los 
aplicación del conocimiento em la investigación, em las prácticas de cooperación o em los proyectos de igualdad.

También podría ayudar a responder a las preguntas sobre si la incorporación al mercado laboral de las expertas em gênero y desarrollo se há traducido em una promoción professional dentro de la cooperación internacional o em otras instituciones donde las mujeres desde SUS posiciones estratégicas de toma de decisiones inciden em los câmbios políticos, institucionales y estructural e as favor de la igualdad de género.

Es enorme el papel de las universidades, como generadoras de conocimiento y especialización de recursos humanos altamente cualificados. Sin embargo, requierede cierta reflexión crítica y reformas profundas desde uma perspectiva de género, si se desea confluir en las transformaciones de sus estructuras de poder, así como el encuentro con um diálogo constructivo em términos feministas en las distintas disciplinas.

En definitiva, se evidencia, la falta de conexión entre la academiay el mundo que la rodea, o dicho de otra manera, com la realidade social y profesional y el objetivo de generar propuestas viables de cambio para la igualad, haciéndose partícipe de las mismas. Por ello, es imprescindible transformare inovar la manera de concebir y transmitir el conocimiento, incorporando las aportaciones realizadas desde los EGED e incorporando como reto la retroalimentación entre la teoría y la práctica. Desde sus inicios, la pretensión de trabajar la teoría desde una experiencia práctica se deriva del propósito de tratar deforma simultânea conocimientosy câmbios socialesparala consecución de la igualdad de género em los contextos particulares del desarrollo.

Esta iniciativa pretendia respondera um desafío, aún presente em la academia, como es el deunir la teoría y la práctica, correspondientes al feminismo y al desarrollo como campos de conocimiento. En este sentido, se observa por um lado, que las académicas, por norma general trabajan en el ámbito de la teoría feminista, pero no pose em experiencia em terreno o trabajo en procesos de desarrollo con enfoque de género. Al igual que Professional ES com uma extensa experiência en género y desarrollo, no someten a esta al a reflexión critico-analítica, ni se nutren de las aportaciones feministas y mucho menos la ponen al servicio de la producción científica.

Por consiguiente, partiendo del conocimiento académico, junto a la experiencia de la práctica, se detecta la necesidad de construir herramientas teóricas y analíticas específicas para poder corregir esta debilidade y unir la teoria com la práctica, com el finde oferecer un mejor nivel de comprensión y capacidade técnica en GED. Por tanto, como reto de los EGED, se contempla la búsqueda y registro de mecanismos adecuados y aceptados localmente, extraídos de la diversidad y pluralidad de experiências que desafían el poder dominante, verificando em la práctica las suposiciones que prevalecen em las políticasde igualdad y de GED.

\section{UNIVERSIDAD, DEMOCRATIZACIÓN Y GESTIÓN DEL CONOCIMIENTO: LOS EGED EN EL AVANCE DE LA AGENDA DE DESARROLLO DE LAS MUJERES}


estructuras patriarcales que operan al interior de las propias universidades. $Y$ aunque los estudios de género han ido ganando un espacio y legitimidad, hasta convertirse en una disciplina académica propia, aún se encuentran en los márgenes de lo que se considera propiamente conocimiento científico.

La tradición académica androcéntrica predominante tiene múltiples impactos, identificándose una falta de apoyo y financiación por parte del androcentrismo del conocimiento que se manifiesta en todas sus expresiones, convirtiéndose en un aliado perfecto en el retroceso y vulneración de los derechos de las mujeres. Aunque hasta el momento, el papel de las universidades como actores del desarrollo ha ido siendo cada vez más importante, posibilitando la presencia de los EGED y el diseño de políticas públicas y aplicación en el terreno mediante programas y proyectos de cooperación con perspectiva de género.

Así, se entienden elementos clave para el fortalecimiento y consolidación de la CUD con perspectiva de género, la normativa e instrumentos en los que se haga referencia expresa a la igualdad de género y la lucha contra la discriminación, como es el caso del Código de Conducta en materia de CUD de las universidades españolas.

En relación a la democratización y gestión del conocimiento, se enfatiza la importancia del fortalecimiento y análisis de experiencias inclusivas y de aquellas que muestran la retroalimentación entre la práctica y la teoría de la mano de la búsqueda de nuevas formas de transferencia y de su intercambio. Las universidades o centros de estudios han de apoyar otras metodologías para incorporar los saberes de mujeres indígenas, afrodescendientes y campesinas.

Y se subraya la implementación de procesos formativos participativos y populares que generan pactos para lograr el empoderamiento de las mujeres. También destacan, la necesidad de repensar los procesos formativos en EGED, dirigiendo el foco de mira hacia espacios donde se acoja a un pensamiento crítico y feminista y facilite el uso de las nuevas tecnologías y de los medios de comunicación.

Actualmente la democratización del conocimiento, va acompañada de un aprendizaje inclusivo, colaborativo y crítico constructivo, a través de instrumentos tales como campañas mediáticas o de incidencia política, cabildeo, mass media etc. con un gran impacto en la sociedad en su conjunto. Así, la incidencia en la sociedad y en las políticas públicas, pasa asimismo por la búsqueda de espacios en los que apenas están representadas las mujeres, como son los medios de comunicación y las redes sociales. Para ello, las nuevas tecnologías de la información y la comunicación resultan herramientas eficaces en la difusión del conocimiento feminista. Aunque no puede obviarse la existencia de una verdadera brecha de género digital, ya que el acceso de las mujeres a las nuevas tecnologías es inferior a la de los hombres, principalmente en los países empobrecidos.

Es importante mencionar que la transferencia de conocimientos e intercambio de información se puede canalizar a través de múltiples canales como las Comunidades de Prácticas, Wikigender u Observatorios de Igualdad etc. codificadas como prácticas prometedoras por su impacto en la gestión del conocimiento. Por tanto, se enfatiza la creación de instrumentos al alcance las organizaciones de mujeres y feministas, académicas y el resto de la sociedad, 
susceptibles de ser empleadas para innovar y mejorar la producción científica, transferencia e intercambio de información y las políticas públicas en pro de la igualdad de género.

\section{ACTUALIZACIÓN FORMATIVA, FINANCIACIÓN Y SOSTENIBILIDAD DE LOS EGED: IGUALDAD Y EFICACIA DE LA AYUDA EN UN CONTEXTO DE CRISIS}

Los EGED han ido evolucionado en consonancia con los cambios vertiginosos producidos en el marco de las políticas internacionales, regionales, estatales y locales vinculadas al desarrollo y a los feminismos. Su implantación en programas académicos o de formación en universidades, institutos de investigación u organizaciones de mujeres, ha supuesto uma oportunidad e impulso para el desarrollo de la disciplina, que se ha ido nutriendo y actualizándose con nuevos contenidos teóricos y metodológicos.

La incorporación de estos estudios, en su mayoría ha sido posible gracias a la existencia de un capital humano constituido por numerosas académicas, activistas feministas y ex alumnas implicadas, que con el tiempo han formado parte y apoyado a centros de estudios e investigación prometedores, como espacios de reflexión, articulación e innovación del conocimiento sobre feminismo y GED (un ejemplo de ello, esel Institute of Development Studies de la Universidadde Sussex, considerado referente mundial en esta materia).

Su producción científica, en ocasiones ha ido por delante de las políticas y las prácticas oficiales de los OOIl y agencias de la cooperaciónal desarrollo, marcando las tendências de la evolución de esta área de conocimiento. Sin embargo, otras veces han sido los grupos de trabajo de los propios OOIl, compuestos por personal académico reconocido, los que han dado um passo más al lá en la construcción teórica y metodológica. Lo certo es que la mayor evolución de la disciplina há sido posible gracias a la cooperación y las alianzas entre los distintos actores: academia, institutos de investigación, organizaciones de mujeres, asociaciones de hombres por la igualdad, OOll, agencias de cooperación, fundaciones, los sindicatos, etc.

Apesar de que los EGED han tenido una evolución constante, algunos programas y cursos de formación llevados a cabo tanto en el estado español como en ALyC, no han podido renovarse com la misma velocidad e incorporar los últimos avance sem la teoría política y práctica de GED. Principalmente, la falta de recursos económicos o humanos especializados, han supuesto la imposibilidad de profundizar en temas emergentes de interes para los debates feministas actuales.

Tal es el caso de las TICs, nuevas masculinidades, feminismos postcoloniales o las aportaciones feministas de la crítica queer y transgéneroen el desarrollo, que entre otras deben formar parte de los debates intelectuales feministas y de los EGED. Por consiguiente, la actualización de los programas GED requiere además de uma adecuación a las necessidades del mundo profesional, uma innovación permanente de las teorias según la evolución política, económica y social.

Por otro lado, se señala como uno de los mayores desafíos de los EGED la financiación, sostenibilidad y autonomía, tanto para el estado español como para ALyC. Apesar de que estos se consideran uma herramienta essencial para avanzar 
em los objetivos de la igualdad de gênero y forman parte de las denominadas buenas prácticas, encuanto alem poderamiento de las mujeres el apoyo institucional econômico no si empreha estado garantizado de modo adecuado para su continuidad.

Es más, aunque en momentos de crisis, la sostenibilidad de los proyectos há ya podido prolongarse por el emprendimiento y voluntad de determinadas académicas o activistas feministas, no obstante esta solución ha sido superficial y finalmente no se ha asegurado su institucionalización. Por ello, para lograr la deseada sostenibilidad alargo plazo, ES necesario fortalecer los mecanismos de ayuda y financiación, garantizando supermanencia.

La crisis económica y sus derivadas políticas macroeconómicas están teniendo un impacto en los cursos de formación o proyectos de investigación en GED,en España y en ALyC. Se evidencian las dificultades particulares de algunos países de la región, vinculados a una pobreza más agudizada junto a los elevados costes de educación, por lo que es particularmente importante assegurar que ésta este alalcance de todas las personas, especialmente de las mujeres y niñas y grupos minoritário sem exclusión social. Debido a la vinculación de los EGED com los interesses de la cooperación internacional, se plantea como uma solución, apoyar este tipo de iniciativas, através de fondos específicos dela AOD. No obstante, se hace referencia también al apoyo procedente de otros fondos públicos o privados, como el de otras instituciones, fundaciones, asociaciones, empresas, centros de formación, universidades etc.

De este modo, con la finalidad de potenciar los resultados y oferecer uma oportunidade para la sostenibilidad de los programas em cuestión, se señalan varios ejemplos a tener en cuenta como: la aplicación de las acciones positivas para corregir la desigualdad de partida em la representación de los grupos desfavorecidos; y el sistema de becas de estúdio o de subvenciones de costos reales de las matrículas para garantizar la participación de las personas que no pueden financiarse este tipo de formación por sus propios medios.

También se subrayan, los intercâmbios de conocimientos y experiências a nivel internacional, mediante la movilidad del professorado y alumnado de diversos países. Las becas com proyección internacional pueden causar un mayor impacto que los EGED sobre los países donde se imparte esta formación, e incluso sobre los países de donde proviene nova na trabajar las personas adscritas a este tipo de programas. En este sentido, se evidencia que las personas formadas em GED em los centros extranjeros de reconocido prestígios e convierten em um potencial enorme para el desarrollo de SUS propios países y regiones, dado que obtien en um os conocimientos privilegiados y aprendizajes basados em su propia experiência de convivência em contextosdiferentes, lo que lês dota de nuevas capacidades y habilidades muy útiles.

Eso ES especialmente importante em el caso de las mujeres, dada su situación de desventaja em el mercado laboral, pues se confirma la mejora de sus oportunidades de empleo y promoción profesional. Por tanto, se constata que la formación em el extranjero no solo permite revertir los conocimientos al regresso a sus países, sino que ademásse adquiere, gracias al título universitario, un mayor prestigio y reconocimiento para aceder a mejores puestos de trabajo, más cualificados y com mejores condiciones laborales. 


\section{RECOMENDACIONES PARA LA MEJORA DE LOS EGED Y LA GESTIÓN DEL CONOCIMIENTO CON PERSPECTIVA DE GÉNERO}

A continuación se muestra un listado de recomendaciones en EGED para la mejora de las acciones en el ámbito de género y gestión del conocimiento:

- Reconocer los EGED como una disciplina de conocimiento, flexible y transformadora generada desde los distintos contextos del desarrollo con un corpusteórico, metodológico y categorial ad hoc.

- Valorar la contribución de los feminismos a los EGED y su vinculación con los movimientos sociales y organizaciones de mujeres.

- Apoyar la creación de nuevas líneas de investigación en GED y la innovación de la producción científica, así como la transferencia de conocimientos, sensibilización e incidencia política.

- Adaptar los EGED a los cambios en el contexto actual e internacional, incluyendo nuevos contenidos, propuestas metodológicas y temas emergentes con el fin de mejorar los resultados en su aplicación y el nivel intelectual y técnico de las personas en formación.

- Identificar a los EGED como una herramienta eficaz para la transversalización del enfoque de género y empoderamiento de las mujeres en las políticas, programas y proyectos de desarrollo.

- Articular la "teoría y la práctica" del feminismo y del desarrollo através del acercamiento entre Academia y el resto de los actores, con la finalidad de potenciar la simbiosisentre los conocimientos adquiridos en "terreno" y la producción científica y cognoscitiva de la Academia.

- Apostar por una "Academia militante y descolonizada" comprometida con la sociedad en su conjunto, en la producción, difusión del conocimiento y formación de agentes de cambio a favor de los procesos de transformación e igualdad de género.

- Replantear en el seno de los EGED la relación compleja entre la diversidad cultural, los feminismos y el desarrollo, identificando las relaciones de poder y el impacto de las mismas -propias de los sistemas dominantes- incluso en las prácticas de integración del enfoque GED.

- Facilitar la participación y acceso a los EGED, de las mujeres pertenecientes a grupos de mayor exclusión social, discriminación y culturas diversas, en la producción de conocimientos.

- Favorecer la generación de alianzas y el "diálogo de saberes" especialmente desde la Academia, centros de estudios, investigación y capacitación con el resto de los actores de la cooperación para el avance de los EGED.

- Fortalecer la institucionalidad del enfoque GED y el establecimiento de políticas de igualdad de género en las universidades, en la lucha contra el androcentrismo del conocimiento y la reproducción de las desigualdades en todas sus manifestaciones.

- Resaltar el papel de las universidades como actores claves de la 
transferencia, intercambio y difusión de la información para la mejora de las políticas GED.

- Incentivar a los EGED, en lo relativo a la contribución de generación de conocimiento y de investigación aplicada y acción participativa (IAP) en base a la complementariedad académica y los saberes no formales vivenciales y cotidianos de las mujeres.

- Poner en valor en el itinerario formativo de los EGED los aprendizajes y las "buenas y malas prácticas" en GED, así como la creación de indicadores desagregados por sexo, mecanismos de transparencia (accountability) y estrategias de incidencia política.

- Facilitar la institucionalización de la Cooperación Universitaria al Desarrollo (CUD) con perspectiva de género y la creación de Redes de EGED en respuesta a un compromiso solidario y para la obtención de recursos humanos especializados al servicio de la sociedad.

- Garantizar el apoyo institucional y económico para lograr la sostenibilidad y autonomía de los EGED sin depender de buenas voluntadespersonales.

- Idear mecanismos de ayuda y financiación para garantizar el acceso a los EGED de todas las personas, sobre todo de mujeres discriminadas $y$ empobrecidas.

- Analizar el impacto y resultados de los EGED y potenciar las experiencias exitosas o "buenas prácticas" para crear mecanismos de financiación y gestión a largo plazo, asegurando su sostenibilidad, especialmente en momentos de crisis. 


\title{
Learning on gender and development studies (EGED): Systematization proposals for improving the shares in Spain
}

\begin{abstract}
Specialized training and research are very important elements for Gender and Development Studies. Learnings and systematizations will be used as a key tool for promoting equality in development cooperation programs and activities. The analysis will be based on the result of I and II Congress celebrated in Latin America and Caribe Region and Spain, strengthening the links in the field of "Gender, Knowledge Management and Development Quality and Effectiveness". Some aspects will also be highlighted to facilitate the political dialogue between different stakeholders focusing in the Academy to further harmonization and effective implementation in gender equality and human rights in the fight against poverty towards a sustainable and inclusive human development.
\end{abstract}

KEY WORDS: Gender and Development Studies, Knowledge Management, Learnings, harmonization between actors and Quality and Effectiveness Development. 


\section{Aprendizagem sobre o estudo de gênero e desenvolvimento (EGED): Propostas de sistematização para a melhoria das ações na Espanha}

\section{RESUMO}

A formação e a investigação especializada são elementos chave para a criação dos elementos de Estudos de gênero e Desenvolvimento (EGED). Os aprendizados sistematizados servirão como um instrumento fundamental para a promoção da igualdade nos programas e acções de cooperação para o desenvolvimento, resultado da I e II Congresso de Estudos sobre Gênero e Desenvolvimento (LAC-Espanha). A análise irá fortalecer os laços em matéria de "Gênero, Gestão do Conhecimento e Eficácia e Desenvolvimento da Qualidade", facilitando o diálogo político entre os vários atores para uma maior harmonização, a implementação efectiva e real da igualdade de género e dos direitos humanos além da capacitação das mulheres na luta contra a pobreza para o desenvolvimento humano sustentável e inclusivo.

PALAVRAS-CHAVE: Estudo de Gênero em Desenvolvimento (EGED). Gestão do Conhecimento. Aprendizagem, a harmonização entre atores e Eficácia e Qualidade do Desenvolvimento. 
ARIAS, S.; MOLINA, E.Universidad y Cooperación al Desarrollo. La experiencia de las universidades de la Ciudad de Madrid. Catarata, Madrid. 2008.

ARIAS, S.; MOLINA, E. Universidad y Cooperación al Desarrollo. Colección Cuadernos Solidarios nำ1-UAM Ediciones, Madrid.

ARNOLD, D. SPEDDING, A. Género, etnicidad y clases sociales. La mujer en los movimientos sociales y movimientos de mujeres. Acción Colectiva y Poder Político, El Viejo Topo, Madrid. 2007.

BERGMAN, S. Análisis de la incorporación de la perspectiva de género en la Cooperación Española. Estudio de caso del Programa de Formación en Cooperación Internacional "Mujeres y Desarrollo". Tesina de Magister en Género y Desarrollo, ICEI Universidad Complutense de Madrid (UCM). 2006.

BUSTELO, M.La evaluación de las políticas de igualdad de género en España. La Catarata, Madrid. 2008.

ESCOBAR, A. Encountering Development.The Making and Unmaking of the Third World, Princeton University Press, Princeton.1995.

JARA,0.Para sistematizar experiencias: una propuesta teórica y práctica. Alforja, Costa Rica, San José. 1994.

JARA, O. Dilemas y desafíos de la sistematización de experiencias. Centro de estudios y Publicaciones Alforja. 2001. Disponible em:

<http://estrategiadidactica.files.wordpress.com/2012/09/jara-oscar dilemas-ydesafc3ados-de-la-sistematizacic3b3n-de-experiencias.pdf>.Acceso 16 febrero 2014.

JOLLY, S. Género y cambio cultural.Institute of Development Study. University of Sussex, Sussex. 2002.

LAGARDE, M. Género y feminism. Desarrollo humano y democracia.Cuadernos Inacabados n25, Horas y horas (Ed), Madrid. 1997.

LEYRA, B.; PÉREZ, A.M.Integración del enfoque de género en políticas, planes y 
MOLINA, M.; SAN MIGUEL, N. Estudios en Género y Desarrollo: Balance y propuestas. Colección Cuadernos Solidarios no5-UAM Ediciones,Madrid. 2009.

MOLINA, M.; SAN MIGUEL, N.(2009). Nuevas líneas de investigación en Género y Desarrollo. Colección Cuadernos Solidarios n3-UAM Ediciones, Madrid. 2009.

MURGUIALDAY, C. Un encuentro urgente: la cooperación universitaria al desarrollo y el trabajo por la equidad de género. En MOLINA M; SAN MIGUEL, N. (eds). Nuevas líneas de investigación en Género y Desarrollo, UAM Ediciones, Madrid, pp-387-405. 2009.

PERONA, P. Globalización, género y desarrollo: pasado y apuntes para el futuro. AECID, Madrid. 2006.

SAN MIGUEL, N. Oportunidades y propuestas en la Agenda de Género y Eficacia. Avanzando hacia 2015. En MOLINA M; SAN MIGUEL. N. (eds). Nuevas líneas de investigación en Género y Desarrollo, UAM Ediciones, Madrid, pp-31-73. 2009.

SEM, A.Development as Freedom. Oxford University Press, UK. 1999.

STRZELECKA, E.Perspectiva cultural en la construcción teórica del desarrollo. En GUARDIOLA, J.; GAGLIARDINI, G. Economía y desarrollo humano: visiones desde distintas disciplinas, Universidad de Granada, Granada. 2009.

Recebido: 10 mai. 2016

Aprovado: 03 ago. 2016

DOI: $10.3895 /$ rbpd.v5n3.4439

Como citar: BAYÓN, M. E. M. Aprendizajes sobre estudios de género y desarrollo (EGED): De la

sistematización a las propuestas para la mejora de las acciones en España. R. bras. Planej.

Desenv.,Curitiba, v. 5, n. 3, p. 341-364, set./dez. 2016. Disponível em: <https://periodicos.utfpr.edu.br/rbpd>

Acesso em: XXX.

Correspondência:

Maria Estefanía Molina Bayón

Ciudad Universitaria de Cantoblanco, 28049 Madrid, España

Direito autoral: Este artigo está licenciado sob os termos da Licença CreativeCommons-Atribuição 4.0

Internacional. 